\title{
Dysmorphic features, simplified gyral pattern and $7 q 11.23$ duplication reciprocal to the Williams-Beuren deletion
}

\author{
Claudia Torniero ${ }^{1}$, Bernardo Dalla Bernardina ${ }^{1}$, Francesca Novara ${ }^{2}$, Roberto Cerini ${ }^{3}$, \\ Clara Bonaglia $^{4}$, Tiziano Pramparo ${ }^{2}$, Roberto Ciccone ${ }^{2}$, Renzo Guerrini ${ }^{5}$ and \\ Orsetta Zuffardi ${ }^{*, 2,6}$
}

\begin{abstract}
${ }^{1}$ Servizio Neuropsichiatria Infantile, Policlinico GB Rossi, Università di Verona, Verona, Italy; ${ }^{2}$ Biologia Generale e Genetica Medica, Università di Pavia, Pavia, Italy; ${ }^{3}$ Istituto di Radiologia, Policlinico GB Rossi, Università di Verona, Verona, Italy; ${ }^{4}$ IRCCS Eugenio Medea, Bosisio Parini-Lecco, Italy; ${ }^{5}$ Azienda Ospedaliero-Universitaria A Meyer, Clinica di Neurologia Pediatrica, Firenze, Italy; ${ }^{6}$ IRCCS Policlinico San Matteo, Pavia, Italy
\end{abstract}

We report a patient with mild pachygyria, ascertained during a screening of subjects with abnormal neuronal migration and/or epilepsy, having a 7q11.23 duplication reciprocal to the Williams-Beuren critical region (WBCR) deletion. He exhibited speech delay and mental retardation together to type II trigonocephaly and other abnormalities. The proband's mother carried the same imbalance, though her phenotype was milder and no abnormal conformation of the cranium was reported. She had suffered a few seizures in infancy, as already described in other duplicated subjects. This genomic imbalance, now described in 17 subjects, including one parent for each of the four probands, is associated with a variable phenotype. Speech impairment is present in most cases; no distinctive facial gestalt is recognizable; seizures have been reported in four subjects and brain magnetic resonance, performed in eight cases, resulted abnormal in six, while detected abnormal neuronal migration in two. Although the clinical description of additional cases is needed to delineate a definite phenotypic core for WBCR duplications, trigonocephaly, also reported in another $\operatorname{dup}(7)(q 11.23)$ patient, is possibly a trait that, together with speech impairment, may call for clinically oriented specific screening. Abnormal development of the cerebral cortex, reported also in the Williams-Beuren deletion, suggests that at least one gene is present in the critical region whose deletion/duplication impairs neuronal migration.

European Journal of Human Genetics (2008) 16, 880-887; doi:10.1038/ejhg.2008.42; published online 12 March 2008

Keywords: 7q11.23 duplication; genotype/phenotype correlations; array-CGH

\section{Introduction}

Speech delay of variable severity appears to be the most common phenotypic manifestation in the 15 cases up to

*Correspondence: Professor O Zuffardi, Biologia Generale e Genetica Medica, Università di Pavia, IRCCS Policlinico San Matteo, Via Forlanini, 14, 27100 Pavia, Italy.

Tel: + 390382 987733; Fax: + 390382525030 ;

E-mail: zuffardi@unipv.it

Received 18 July 2007; revised 22 January 2008; accepted 5 February 2008; published online 12 March 2008 now reported of $7 q 11.23$ duplication reciprocal of the Williams-Beuren deletion. ${ }^{1-6}$ In three instances the duplication was found in either proband's father ${ }^{2}$ or mother (cases 5 and 6 in Ber et al) ${ }^{5}$ who, although briefly described, did not appear to exhibit speech or cognitive impairment, arising the suspicion that the duplication can also be present in seemingly healthy individuals. In an array-CGH screening of 134 patients with cortical malformations and/or epilepsy, we found 2 patients with the 7q11.23 duplication. The first was ascertained for epilepsy ${ }^{6}$ whereas 
the present case was ascertained because of a cortical malformation. The duplication was also present in his mother who exhibited impaired language processing and expression with defective phonologic and articulation skills. Trigonocephaly, present in the proband and in the patient reported by Kriek et $\mathrm{al}^{2}$ may represent a phenotypic trait that, together with speech impairment, might point to the $7 \mathrm{q} 11.23$ duplication.

\section{Clinical report \\ Proband}

The proband, 131/06: case 13 in Table 1 (Figure $1 \mathrm{a}$ and $\mathrm{b}$ ) is the second child of healthy unrelated parents. His sister had normal development. The propositus was born at 40 weeks, after spontaneous delivery, weighing $3060 \mathrm{~g}$ (25th percentile), measuring $50 \mathrm{~cm}$ (25th percentile) in length and with a cranial circumference (OFC) of $35 \mathrm{~cm}$ (50th percentile). Apgar scores were 9 and 10 at 1 and $5 \mathrm{~min}$, respectively. Pilonidal sinus at the very top of the cleft between the buttocks and cryptorchidism were noticed early on. At 6 months of age psychomotor delay was evident: he was not able to roll back to side and front to side and could not wiggle forward on the floor. He was able to hold his head up, to search and play with hands and bring them to mouth; sometimes he had responsive smiling and pronounced extended vowel sounds. Chromosome analysis at 7 months was reported as normal. Clinical examination at 8 months of age revealed mild trigonocephaly, low-set, posteriorly rotated ears, sparse anterior scalp hair, left eye with exotropia, bulbous nasal tip, ogival palate, short philtrum with thin lips, short lingual frenulum, short neck, bilateral pes cavum, severe motor delay and hypotonia. EEG, while awake and during falling asleep, showed unusual rapid beta activity, synchronous over the rolandic and vertex regions. At the age of 14 months the OFC was $47 \mathrm{~cm}$ (50th percentile). He was able to roll over, could sit unsupported and stand with support, although axial hypotonia was still evident. Marked drooling of saliva was present. Eye tracking was poor. He was able to produce bisyllabled sounds and to communicate through pointing. Short lingual frenulum had been surgically corrected. Fundus oculi, audiometric investigations, visual and somesthetic evoked potentials and EEG were normal. Griffith's mental development scale gave a global score of 50 (normal range: 85-100) with subscale A (locomotor) score $<50$, subscale B (personal-social), subscale C (hearing and speech) 56 , subscale D (hand and eye coordination) $<50$. Brain magnetic resonance imaging (Figure 2) showed a simplified gyral pattern, with areas of cortical thickening, slightly open opercular fissures, multiple areas of increased signal in T2 and of reduced signal intensity in $\mathrm{T} 1$ in the subcortical white matter, probably corresponding to dilated perivascular spaces, interspersed with radial migration abnormalities. There were, in addition, poorly formed hippocampi and a markedly hypoplastic vermis. A triangular configuration of the skull was present, in association with a prominent metopic suture and with deformity of the sutures of the anterior-basal part of the skull, consistent with type II trigonocephaly.

\section{Mother}

The mother (1333/06: case 14 in Table 1), 31 years old, was born as the second child of healthy unrelated parents. Her brother was healthy. She had had normal developmental milestones. At 1 year and 2 years 6 months (Figure 1c) she had two epileptic seizures, after which she was given phenobarbital for 3 years. Her EEG was reported as normal. At primary school (Figure 1d), she learned to write and read but was not able to understand simple and logic concepts of mathematics and exhibited memory difficulties. She stopped her studies after the first 8 years of compulsory school. Presently, her stature was $163 \mathrm{~cm}$ (50th percentile), weight $64 \mathrm{~kg}$ (75th percentile). Her expressive language skills were very poor, with simple sentences, phonology deficits and defective articulation of speech sounds. Although not formally tested, comprehension appeared to be limited.

\section{Materials and methods}

Molecular karyotyping was performed through array comparative genomic hybridization (array-CGH) with the Agilent kit (Human Genome CGH Microarray, Agilent Technologies, Santa Clara, CA). The array-CGH platform is a 60-mer oligonucleotide-based microarray that allows a genome-wide survey and molecular profiling of genomic aberrations with a resolution of $\sim 100 \mathrm{~kb}$ (kit 44B). To better define the breakpoints of the duplication and to increase the resolution of experiments, a customized array (constituted by 44000 oligomeres) was designed using all possible probes mapped in duplicated region and present in the Agilent catalog (https://earray.chem.agilent.com/ earray/) and adding a bigger number of control probes than those duplicated. In this way, we obtained a resolution of $1 \mathrm{~kb}$ in single copy region. DNA, for all experiments, was extracted from peripheral blood of patients with QIAamp DNA Blood Mini Kit (Qiagen) according to the manufacturer's protocol. The array-CGH experiments have been done according to De Gregori et al (2007). ${ }^{7}$ The array was analyzed through the Agilent scanner and the Feature Extraction software (v9.1). Graphical overview was obtained using the CGH analytics software (v3.4.27) according to hg 17 (genome building 35 of May 2004). Genotyping of polymorphic loci on DNAs of proband, mother and paternal and maternal grandparents was performed by amplification with primers labeled with fluorescent probes (ABI 6-Fam and 8-Hex) followed by analysis on an ABI 3100 Genetic Analyzer (Applied Biosystems). The primers for polymorphic loci were chosen 


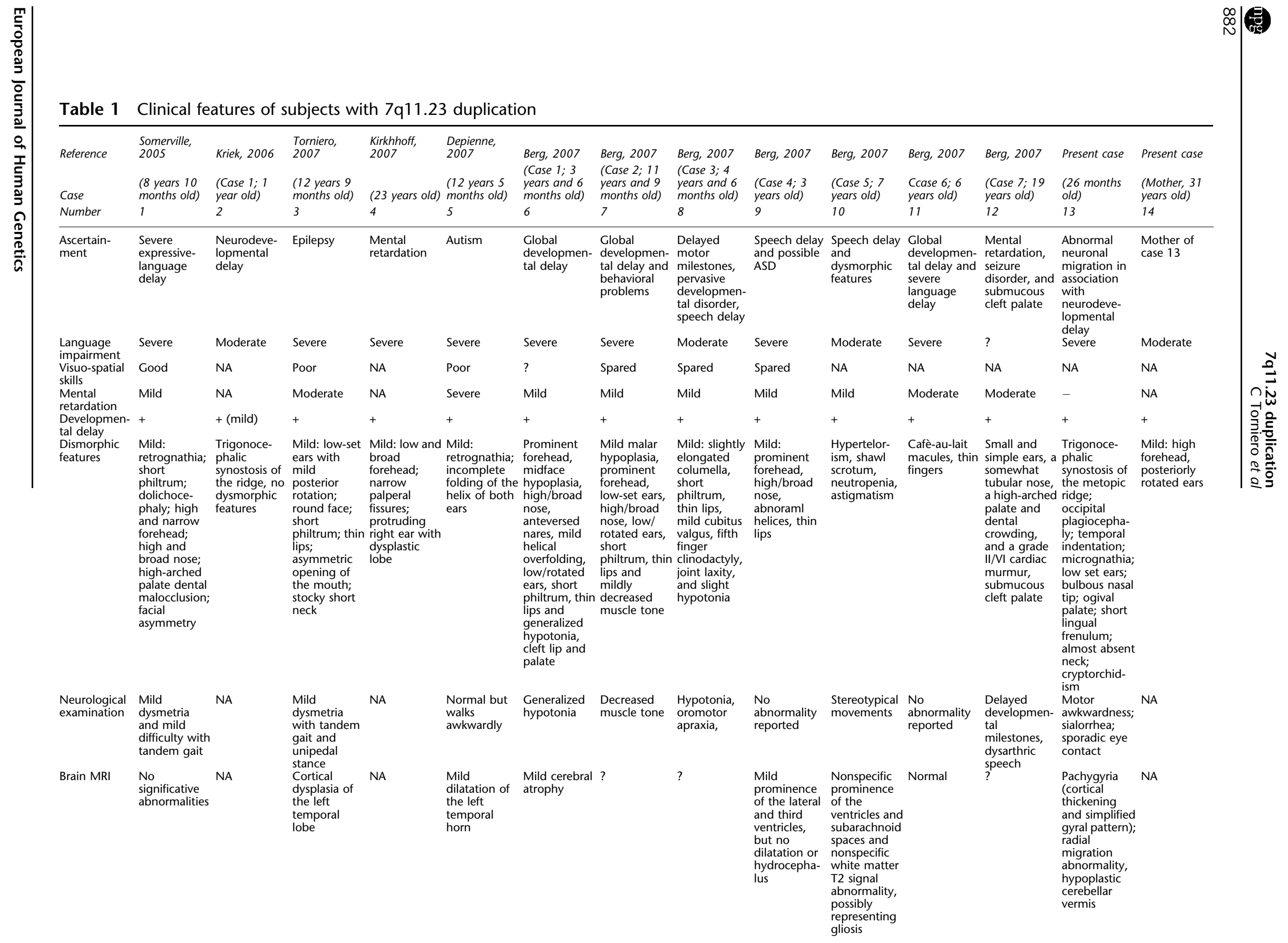




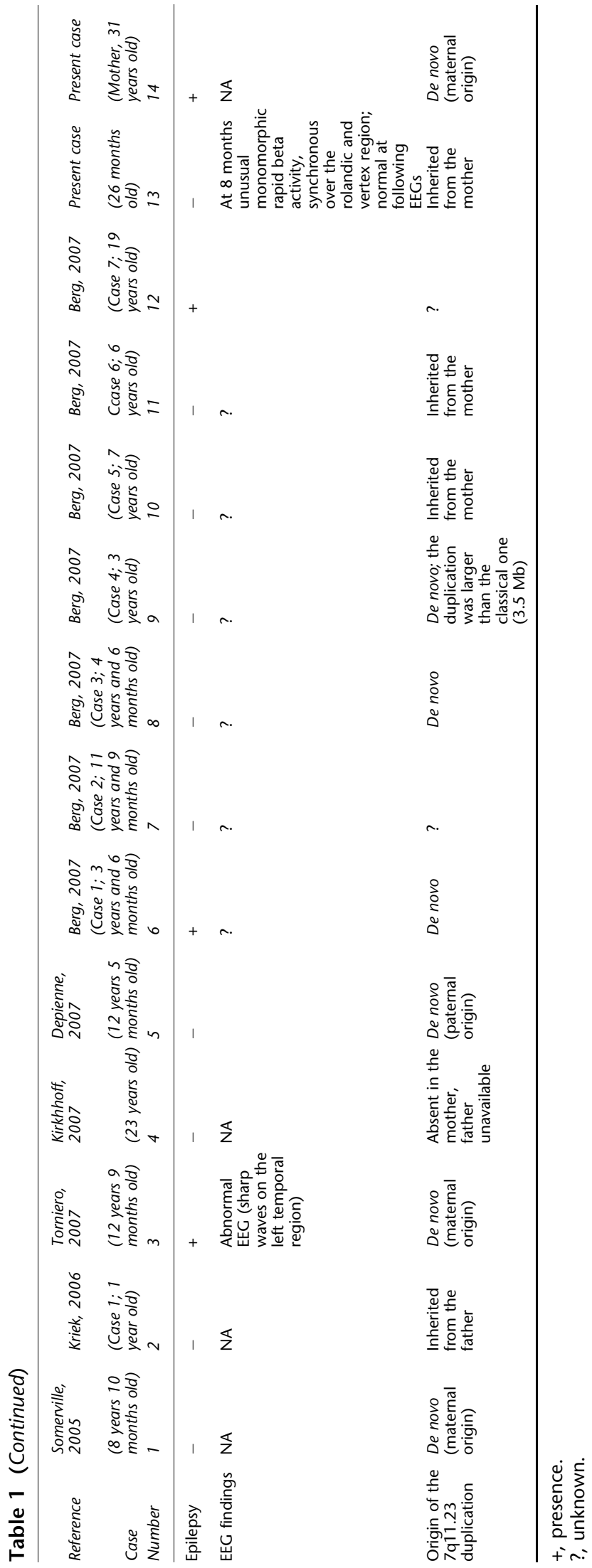

among those known and present in the site of UCSC Genome Browser (http://genome.ucsc.edu/). Additional primers were designed by searching dinucleotide repeats, with the highest number of copies within the duplicated 7q11.23 region, using the database tool Tandem Repeats Finder (http://tandem.bu.edu/trf/trf.intermediate. submit.html). The primers for these unlisted polymorphic dinucleotides were designed using Primer 3 Input (http:// frodo.wi.mit.edu/cgi-bin/primer3/primer3_www.cgi). FISH was performed on metaphase and interphase cells from the patient, his mother and maternal grandmother to investigate if the duplication was direct or inverted and if it was mediated by a paracentric inversion. ${ }^{8}$ To this purpose, we used the following BAC clones: RP11-815K13 proximal to the critical region, CTA-208H19 and RP5- 1186P10, both within the critical duplicated region. DNA extraction, labeling, hybridization and detection and FISH experiments were undertaken, as described previously. ${ }^{9}$ We also performed analysis of the breakpoints with self-chain and segmental duplications repeats tools from UCSC Genome Bioinformatics (http://genome.ucsc.edu/).

\section{Results}

Whole genome array-CGH with a resolution of about $100 \mathrm{~kb}$ revealed a duplication in the proband of at least 1 . $718 \mathrm{Mb}$ ranging from 71.865 to $73.583 \mathrm{Mb}$, flanked by oligomeres at 71.846 and $73.926 \mathrm{Mb}$ that were not duplicated (Figures 3a and b). Actually, the abnormal segment included part of the segmental duplications (SDs) flanking the WBS region, whose copy number status is difficult to determine through array-CGH experiments. After exclusion of probes falling into those SDs, the duplication ranged from 72.295 to $73.583 \mathrm{Mb}(1.288 \mathrm{Mb})$. Array-CGH experiment performed on the proband's mother demonstrated that she had the same duplicated region, while her parents did not (cases 1878/06 and 1879/ 06). Several known CNVs were present in proband's and mother's array profiles (4 and 6, respectively); they were all reported in the database of the genomic variants (http:// projects.tcag.ca/variation/) and, obviously, could not be attributed to the proband or the reference DNA (pool of 10 males for the proband and of 10 females for his mother). To better characterize the breakpoints of the duplicated region, a customized-array platform, with specific oligomeres for $7 \mathrm{q} 11.23$ region and control oligomeres localized in different chromosomes, was drawn allowing a resolution of about $1 \mathrm{~kb}$ in single copy regions. Excluding the SDs blocks flanking the duplication, the array-CGH experiment revealed that the duplicated region ranged from 72.276 to $73.586 \mathrm{Mb}$ (Figure 3c) and it was of at least $1.31 \mathrm{Mb}$. Microsatellite analysis performed on proband, parents and maternal grandparents showed the rearrangement to have occurred at the grandmother's meiosis. In fact, the 

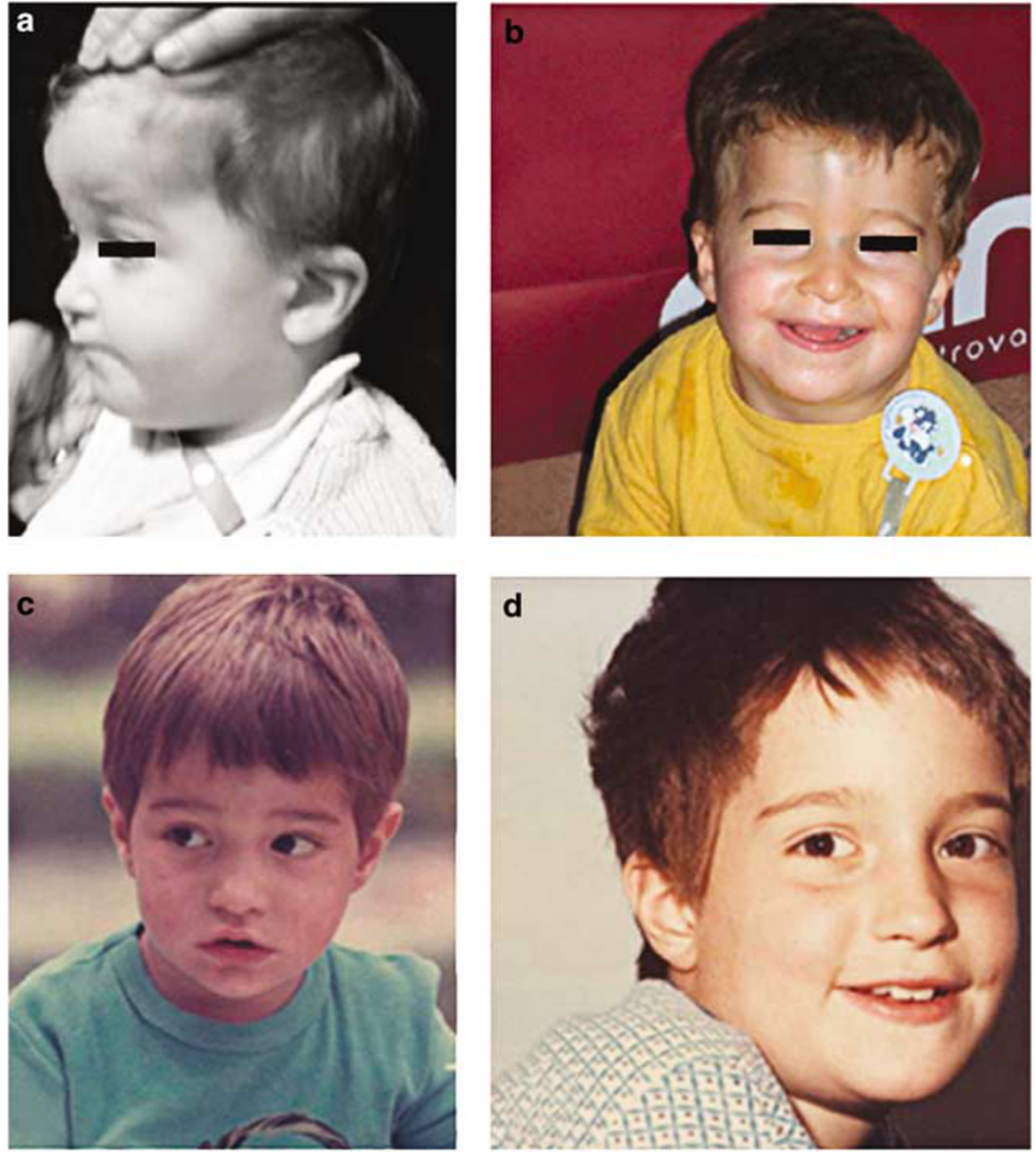

Figure $1(\mathbf{a}, \mathbf{b})$ The proband at 26 months of age showing the prominence of the metopic suture and narrowing of bitemporal distance; the mother at 2 (c) and 7 (d) years of age.
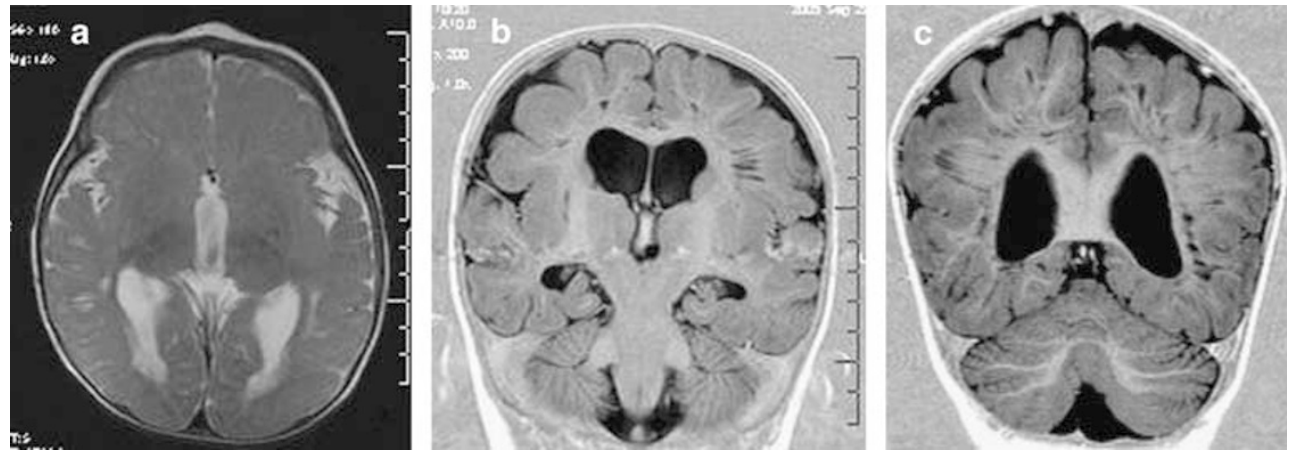

Figure 2 Magnetic resonance imaging of the brain. (a) T2 weighted axial image. Note the prominence in the frontal midline, corresponding to the metopic suture. Areas of increased signal of the white matter are visible near the occipital horns. (b) T1 weighted coronal image. Note thickening of the cerebral cortex in the parietal lobes, especially on the right, with an overlying simplified gyral pattern. The caliper on the right side of the image measures $1 \mathrm{~cm}$; cortical thickness at the level of the parietal lobes and insular cortex is above $1.5 \mathrm{~cm}$, while thickness of he normal cortex is around $0.4 \mathrm{~cm}$. Some cystic areas are visible in the subcortical white matter just beneath the left parietal lobe. (c) T1 weighted coronal image, taken more caudally with respect to (b). Radially disposed cystic areas span from the lateral ventricular walls to the subcortex in the white matter. They most likely represent columns of radial migration abnormality.

proband's mother had three alleles, two maternal and one paternal at D7S2476 (Figure 3d) and STS3_dup7q. ArrayCGH has also been performed in grandparents' DNAs confirming the absence of 7q11.23 duplication. FISH analysis, in both the proband and in his mother, demonstrated that the duplication was in tandem (Figure 3e). 


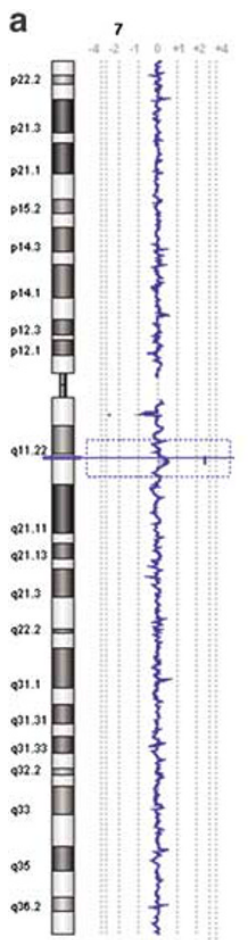

d

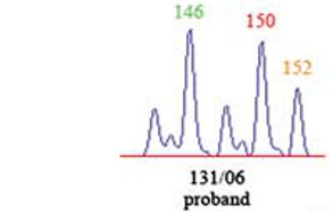

b $7: 71043917.74960661,3.91 \mathrm{Mb}$

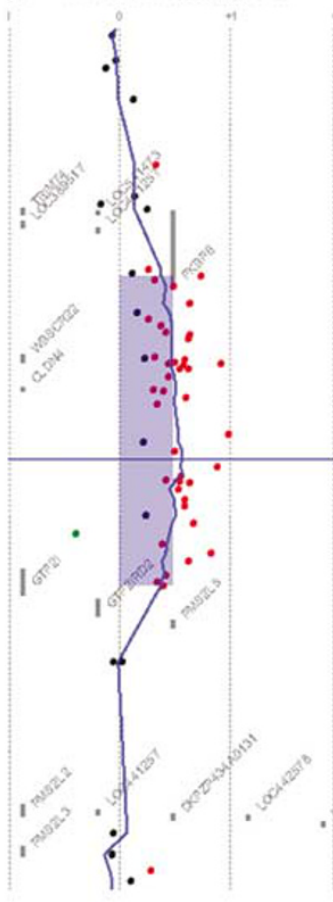

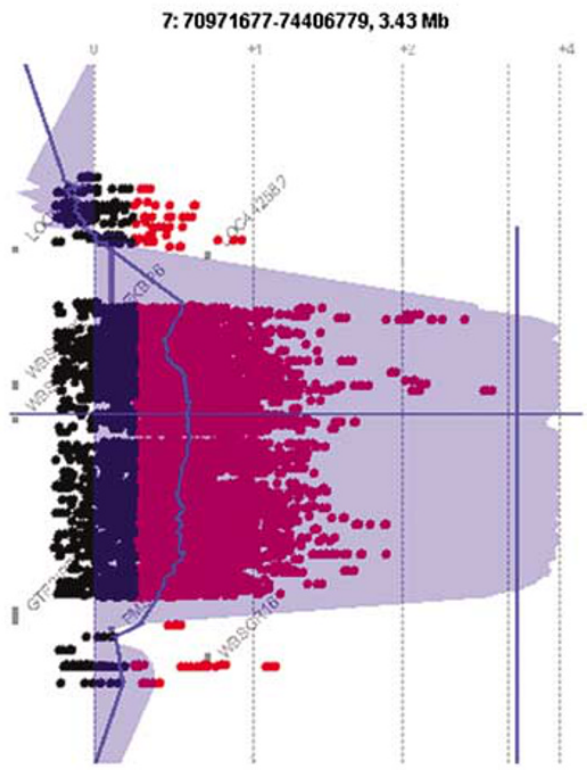

e

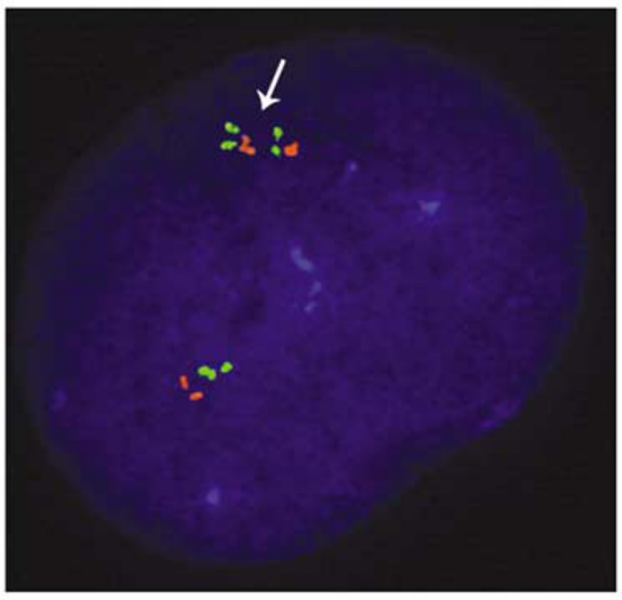

Figure 3 (a) Chromosome 7 profile of the $44 \mathrm{k}$ array (about $100 \mathrm{~kb}$ resolution) and its enlargement (b) showing the $7 \mathrm{q} 11.23$ duplication (red spots) found in the proband and his mother; in gray are listed some of the genes included in the shown region (from 71.043 to $74.960 \mathrm{Mb}$ ); (c) profile of the customized array at the final resolution of $1 \mathrm{~kb}$ in single copy regions showing that the dup (7)(q11.23) covers the Williams-Beuren classical deletion of about $1.3 \mathrm{Mb}$; (d) D7S2476 microsatellite analysis of DNAs from the proband, his parents and maternal grandparents showing the grandmaternal origin of duplication; (e) interphase FISH in proband's lymphocytes with CTA-208H19 and RP5-1186P10, both within Williams-Beuren critical region (red and green, respectively) showing that the duplication (white arrow) is direct.

\section{Discussion}

To our knowledge 15 cases of 7q11.23 duplication, reciprocal to the Williams-Beuren deletion syndrome, have been published. Based on available descriptions, no clearly defined phenotype can be attributed to WBCR duplication, making clinically oriented specific screening unreliable. Amongst the previous descriptions, ascertainment had been prompted for different reasons (Table 1) ranging from developmental and language delay to autism. Both our cases (cases 3 and 13) belong to a cohort of individuals 
analyzed by array-CGH because of anomalous cerebral cortex development and/or epilepsy. In 11 of the reported cases, parents were also analyzed for the presence/absence of the rearrangement. The finding that four of the parents (cases 2, 10, 11 and 13) had the same duplication present in their offspring but a relatively normal phenotype points to phenotypic variability. Retrospectively, the mother of the patient reported herein (case 14) had in fact poor language skills whereas no details were reported about the cognitive status of the father of case 2 and the mother of case 10 . The mother of case 11 exhibited no developmental delay and was able to complete high school in a regular classroom setting. This observation recalls what can be observed in the 22q11 deletion syndrome for which different definitions have been used, such as DiGeorge syndrome, velocardiofacial syndrome, conotruncal anomaly face, Cayler syndrome and Opitz GBB syndrome and different diagnoses have at times been made, even in different members of the same family segregating the same deletion. $^{10}$

Mild trigonocephaly was a phenotypic feature of our patient. Trigonocephalic synostosis of the metopic ridge had been described in only 1 out of 17 reported patients with the same duplication (case 2). In addition, plagiocephaly was reported in one out of three cases with supernumerary ring chromosomes, all mosaic, including the WBS region. ${ }^{11,12}$ Trigonocephaly might be either coincidental with the WBCR duplication or resulted from a duplicated dosage-sensitive gene within the critical region, whose effects act with low penetrance. Since trigonocephaly is exceedingly rare (1:10000-15000), ${ }^{13}$ and assuming that the frequency of WBCR duplication is the same of the deletion $(1 / 20000)$ or even lower, ${ }^{14}$ the probability for the two events to co-occur is about 1:200000000. Clinical variability between family members carrying the same mutation for one of the known genes that have been associated with trigonocephaly represents a major challenge for genetic counseling. ${ }^{15}$

Although the clinical risk for trigonocephaly associated with WBCR duplication is likely to be relatively low, in our opinion, association of this phenotypic trait with poor speech development should prompt a search of the 7q11.23 duplication. Magnetic resonance imaging of the brain, performed in the case reported by Torniero et $a l^{6}$ and in the child described in this report, revealed abnormal development of the cerebral cortex, which is likely to contribute to cognitive and language impairment. In particular, Torniero's case exhibited cortical thickening and an abnormal gyral pattern in the left sylvian and temporal cortex. In the patient described here, similar abnormalities were more widely distributed, bilaterally involving the parieto-occipital cortex. Additional abnormalities were also noted, including multiple cystic areas within the subcortical white matter in the parieto-occipital cortex, interspersed with radial white matter fibers, consistent with radial migration abnormality. Cortical abnormalities consisting in mild cerebral atrophy were also reported in case 6 , although atrophy is an aspecific term and not clearly related to prenatal or postnatal events. Gyral pattern abnormalities in individuals with Williams syndrome have been reported possibly contributing to the distinct cognitive and behavioral profile accompanying the disorder. ${ }^{16-19}$ These findings point to the presence in the critical region of at least a gene whose deletion/ duplication impairs neuronal migration, to an extent that may be detected or not using MRI.

\section{Conclusion}

The two patients we are reporting in this paper confirm the phenotypic variability associated with the $7 \mathrm{q}$ duplication reciprocal to the Williams-Beuren deletion. We found two such cases (cases 3 and 13 in Table 1) among 134 patients analyzed for abnormal neuronal migration and/or epilepsy. These series of patients represent, in turn, a subset of 510 individuals we analyzed for idiopathic mental retardation with or without associated dysmorphisms or congenital malformations. Thus, the frequency of the WBCR duplication is $0.39 \%$ of the whole sample we examined and is four times higher in the sample with abnormal neuronal migration and/or epilepsy. This figure is strikingly similar to that of WBS deletion estimated from Stevenson et al ${ }^{20}$ in a large cohort of patients with mental retardation, corresponding to $0.31 \%$. However, the presence of the duplication in four healthy or nearly healthy parents of 13 probands suggests that its frequency is even higher and that the duplication might go undetected in some individuals. In fact, in the mother of our proband no cytogenetic abnormality had been suspected in childhood, in spite of her exhibiting an association of seizures and poor school achievement. These findings point to enlarge whole genome investigations not only to individuals with the classical chromosomal phenotype (mental retardation, facial dysmorphisms and/or congenital anomalies) but also to those exhibiting mild cognitive impairment, with or without epilepsy.

\section{Acknowledgements}

This work was supported by the Telethon foundation (to TP and $R G$ : GGP05177) and PRIN 2005, Fondazione Mariani and Fondazione CARIPLO (all to O.Z.). We thank the family for its cooperation.

\section{References}

1 Somerville MJ, Mervis CB, Young EJ et al: Severe expressive language delay related to duplication of the Williams-Beuren locus. N Engl J Med 2005; 353: 1694-1701.

2 Kriek M, White SJ, Szuhai K et al: Copy number variation in regions flanked (or unflanked) by duplicons among patients with developmental delay and/or congenital malformations; detection 
of reciprocal and partial Williams-Beuren duplications. Eur I Hum Genet 2006; 14: 180-189.

3 Depienne C, Heron D, Betancur C et al: Autism, language delay and mental retardation in a patient with $7 \mathrm{q} 11$ duplication. $J$ Med Genet 2007; 44: 452-458.

4 Kirchhoff M, Bisgaard AM, Bryndorf T, Gerdes T: MLPA analysis for a panel of syndromes with mental retardation reveals imbalances in $5.8 \%$ of patients with mental retardation and dysmorphic features, including duplication of the Sotos syndrome and Williams-Beuren syndrome regions. J Med Genet 2007; 50: $33-42$.

5 Berg JS, Brunetti-Pierri N, Peters SU et al: Speech delay and autism spectrum behaviors are frequently associated with duplication of the 7q11.23 Williams-Beuren syndrome region. Genet Med 2007; 9: $427-441$.

6 Torniero C, Dalla Bernardina B, Novara F et al: Cortical dysplasia of the left temporal lobe might explain severe expressivelanguage delay in patients with duplication of the WilliamsBeuren locus. Eur J Hum Genet 2007; 15: 62-67.

7 De Gregori M, Ciccone R, Magini P et al: Cryptic deletions are a common finding in 'balanced' reciprocal and complex chromosome rearrangements: a study of 59 cases. J Med Genet 2007; 44: $750-762$.

8 Bayes M, Magano LF, Rivera N, Perez-Jurado LA: Mutational mechanisms of Williams-Beuren syndrome deletions. Am J Hum Genet 2003; 73: 131-151.

9 Pramparo T, Giglio S, Gregato G et al: Inverted duplications: how many of them are mosaic? Eur J Hum Genet 2004; 12: 713-717.

10 De Decker HP, Lawrenson JB: The $22 \mathrm{q} 11.2$ deletion: from diversity to a single gene theory. Genet Med 2001; 3: 2-5.
11 Chantot-Bastaraud S, Muti C, Pipiras E et al: Clinical findings and cytogenetic analysis of small supernumerary ring chromosomes 7: report of two new cases. Ann Genet 2004; 47: 241-249.

12 Lichtenbelt KD, Hochstenbach R, van Dam WM, Eleveld MJ, Poot M, Beemer FA: Supernumerary ring chromosome 7 mosaicism: case report, investigation of the gene content, and delineation of the phenotype. Am J Med Genet A 2005; 132: 93-100.

13 Lajeunie E, Le Merrer M, Marchac D, Renier D: Syndromal and nonsyndromal primary trigonocephaly: analysis of a series of 237 patients. Am J Med Genet 1998; 75: 211-215.

14 Turner DJ, Miretti M, Rajan D et al: Germline rates of de novo meiotic deletions and duplications causing several genomic disorders. Nat Genet 2008; 40: 90-95.

15 Jehee FS, Alonso LG, Cavalcanti DP et al: Mutational screening of FGFR1, CER1 and CDON in a large cohort of trigonocephalic patients. Cleft Palate Craniofac J 2006; 43: 48-51.

16 Galaburda AM, Bellugi U: V. Multi-level analysis of cortical neuroanatomy in Williams syndrome. J Cogn Neurosci 2000; 12 (Suppl 1): 74-88.

17 Schmitt JE, Watts K, Eliez S, Bellugi U, Galaburda AM, Reiss AL: Increased gyrification in Williams syndrome: evidence using 3D MRI methods. Dev Med Child Neurol 2002; 44: 292-295.

18 Thompson PM, Lee AD, Dutton RA et al: Abnormal cortical complexity and thickness profiles mapped in Williams syndrome. J Neurosci 2005; 25: 4146-4158.

19 Gaser C, Luders E, Thompson PM et al: Increased local gyrification mapped in Williams syndrome. Neuroimage 2006; 33: 46-54

20 Stevenson RE, Procopio-Allen AM, Schroer RJ, Collins JS: Genetic syndromes among individuals with mental retardation. Am J Med Genet A 2003; 123: 29-32. 